\title{
Insulin-Like Growth Factor-1 Inhibits Mature Oligodendrocyte Apoptosis during Primary Demyelination
}

\author{
J. L. Mason, ${ }^{1}$ P. Ye, ${ }^{2}$ K. Suzuki, ${ }^{3}$ A. J. D'Ercole, ${ }^{2}$ and G. K. Matsushima ${ }^{1,4}$ \\ ${ }^{1}$ Curriculum in Neurobiology and University of North Carolina Neuroscience Center, Departments of 2 Pediatrics, \\ ${ }^{3}$ Pathology and Laboratory Medicine, and 4 Microbiology and Immunology and Program in Molecular Biology and \\ Biotechnology, University of North Carolina, Chapel Hill, North Carolina 27599
}

\begin{abstract}
Metabolic insult results in apoptosis and depletion of mature oligodendrocytes during demyelination. To examine the role of insulin-like growth factor-1 (IGF-1) during acute demyelination and remyelination in the adult CNS, we exposed transgenic mice that continuously express IGF-1 (IGF-1 tg) to cuprizone intoxication. Demyelination was observed within the corpus callosum in both wild-type and IGF-1 $\mathrm{tg}$ mice 3 weeks after exposure to cuprizone. Wild-type mice showed significant apoptotic mature oligodendrocytes and a dramatic loss of these cells within the lesion that resulted in near complete depletion and demyelination by week 5 . In contrast, the demyelinated corpus callosum of the IGF-1 tg mice was near full recovery by week 5 . This rapid recovery was apparently caused by survival of the mature oligo-
\end{abstract}

dendrocyte population because apoptosis was negligible, and by week 4 , the mature oligodendrocyte population was completely restored. Furthermore, despite demyelination in both wild-type and IGF-1 tg mice, oligodendrocyte progenitors accumulated only in the absence of mature oligodendrocytes and failed to accumulate if the mature oligodendrocytes remained as demonstrated in the IGF-1 tg mice. These results suggest that IGF-1 may be important in preventing the depletion of mature oligodendrocytes in vivo and thus facilitates an early recovery from demyelination.

Key words: insulin-like growth factor-1; oligodendrocytes; progenitors; myelin; demyelination; apoptosis; mice; transgenic
The terminal fate of demyelinating disorders is thought to be a consequence of oligodendrocyte and/or axonal depletion (Compston, 1996). Previous studies suggest that chronic demyelination results from the depletion of the mature oligodendrocytes within a lesion (Johnson and Ludwin, 1981; Mason et al., 2000) (for review, see Raine, 1997). This depletion of the mature oligodendrocytes appears to be caused by the apoptotic death of these cells (Pender et al., 1991; Taniike et al., 1999; Mason et al., 2000). The inhibition of oligodendrocyte death during primary demyelination, therefore, may be critical in preventing the progression of a demyelinating disease to a chronic state.

Insulin-like growth factor 1 (IGF-1) has been shown to induce myelination in vitro (Mozell and McMorris, 1991) and in vivo (for review, see D'Ercole et al., 1996; Werther et al., 1998) while also protecting mature oligodendrocytes from a pathological insult. IGF-1 has been demonstrated to reduce lesion severity and clinical deficits in experimental autoimmune encephalomyelitis (EAE) (Yao et al., 1995, 1996). Furthermore, IGF-1 promotes the longterm survival of mature oligodendrocytes in culture (Barres et al., 1993 ) and inhibits mature oligodendrocyte apoptosis in vitro (Cho et al., 1997; Ye and D'Ercole, 1999). However, it is not known whether IGF-1 can inhibit mature oligodendrocyte apoptosis in vivo after a demyelinating insult.

In this study, we used a transgenic line of mice expressing high levels of IGF-1 within the brain (IGF-1 tg mice) (Ye et al., 1995) to investigate whether IGF-1 can protect mature oligodendrocytes

Received March 27, 2000; revised May 3, 2000; accepted May 3, 2000.

This work was supported in part by National Institute of Neurological Disorders and Stroke Grants NS35372 (G.K.M.), NS24453 (K.S.), and NS38891 (A.J.D.), and Grant RG2754A1 from the National Multiple Sclerosis Society (G.K.M.), in the University of North Carolina Neuroscience Center receiving core support from National Institute of Child Health and Human Development Grant HD03110. We are grateful to Dr. Robert Bagnell, Victoria Madden, and Clarita Langaman for their assistance with the morphometric analysis.

Correspondence should be addressed to Dr. G. K. Matsushima, University of North Carolina Neuroscience Center, CB\# 7250, University of North Carolina, Chapel Hill, NC 27599. E-mail: gkmats@med.unc.edu.

Dr. Mason's present address: Columbia University College of Physicians and Surgeons, Department of Pathology, New York, NY 10032.

Copyright (C) 2000 Society for Neuroscience $0270-6474 / 00 / 205703-\bullet \$ 15.00 / 0$ from apoptotic death during primary demyelination in vivo. During cuprizone intoxication, acute demyelination occurs within the CNS (Hiremath et al., 1998) that features mature oligodendrocyte apoptosis and subsequent depletion of mature oligodendrocytes from the corpus callosum (Mason et al., 2000). Although acute demyelination was observed in both cuprizone-treated wild-type and $I G F-1$ tg mice, mature oligodendrocyte death was predominantly observed in the wild-type mice and infrequently detected in the $I G F-1 \mathrm{tg}$ mice. Furthermore, an early recovery from demyelination was observed in IGF-1 tg mice. These results suggest that IGF-1 may enhance the recovery from a demyelinating insult presumably by retaining the mature oligodendrocyte population.

\section{MATERIALS AND METHODS}

Induction of demyelination. C57BL/6J wild-type mice (Jackson Laboratory, Bar Harbor, ME) and IGF-1 $\mathrm{tg}$ mice were maintained in our colony. The IGF-1 tg mice express IGF-1 under the mouse metallothionein-I promoter, and these mice were backcrossed more than six generations onto the C57BL/6 background (Ye et al., 1995). At 8 weeks of age, mice were fed a diet containing $0.2 \%$ cuprizone (Sigma, St. Louis, MO) for 5 weeks to induce demyelination, as described previously (Hiremath et al., 1998). Sham and cuprizone-treated mice were killed weekly. The forebrains from the mice were removed for morphological and biochemical analysis as described previously (Coetzee et al., 1996; Ye et al., 1996; Mason et al., 2000). Mice were maintained in sterile pathogen-free conditions under Institutional Animal Care and Use Committee and University of North Carolina Division of Laboratory Animal Medicine guidelines.

Immunohistochemistry. All comparative analyses were focused in the corpus callosum on either side near midline. Frozen $(10 \mu \mathrm{m})$ or paraffinembedded $(5 \mu \mathrm{m})$ brain samples were cut in serial sections between 220 and 260 in the mouse brain atlas (Sidman et al., 1971). Frozen sections were stained either for IGF-1 using a rabbit anti-IGF-1 antibody (a gift from Dr. Underwood, Chapel Hill, NC) or for oligodendrocyte progenitors using a rabbit anti-NG2 antibody (a gift from Dr. Stallcup, San Diego, CA) as described previously (Mason et al., 2000). No positive cell staining was observed in tissue sections incubated with a control isotype-matched antibody (rabbit IgG; Vector Laboratories, Burlingame, CA) in place of the primary antibody.

Paraffin-embedded sections were stained for the $\mathrm{Pi}$ isoform of glutathione- $S$-transferase (GST-Pi; a mature oligodendrocyte marker), GST-Pi/apoptosis (apoptosis was detected using the Neurotacs assay kit from Trevigen), and Ricinus communis agglutin-1 (RCA-1; a marker for microglia/macrophages) as described previously (Hiremath et al., 1998; Morell et al., 1998; Mason et al., 2000).

Cell number quantification. RCA- ${ }^{+}, \mathrm{GST}^{\mathrm{P}} \mathrm{i}^{+}, \mathrm{GST}-\mathrm{Pi} /$ apoptosis $^{+}$, and 

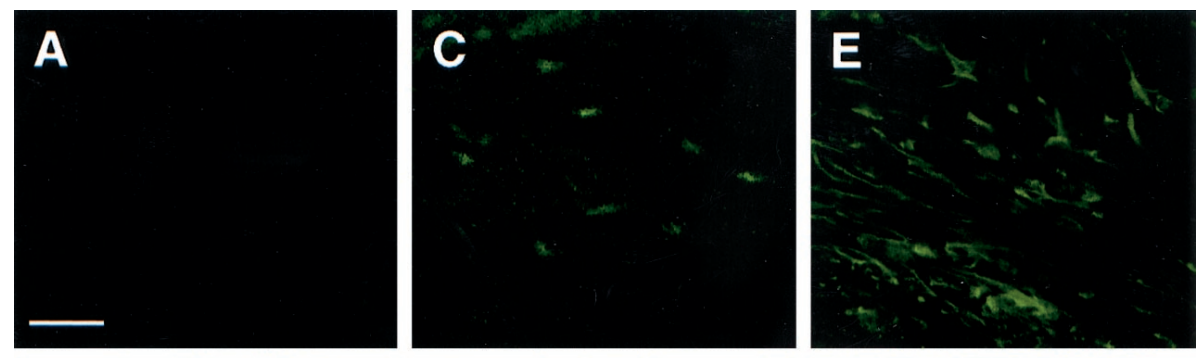

callosum 1 . Presence of IGF-1 within the corpus zone. Frozen brain sections from wild-type $(A, C$, $E)$ and $I G F-1$ tg $(B, D, F)$ mice were immunostained with anti-IGF-1 (green). Although few IGF- $1^{+}$cells were observed within the corpus callosum of the untreated control wild-type mice $(A)$, a substantial number were observed in the $I G F-1$ tg mice $(B)$. At 3 weeks, some $\mathrm{IGF}-1^{+}$cells were present in wild-type mice $(C)$, whereas large numbers appeared in the $I G F-1 \mathrm{tg}$ mice $(D)$. At 5 weeks, a large accumulation of IGF-1 ${ }^{+}$cells was observed in the wild-type mice $(E)$, whereas in $I G F-1 \mathrm{tg}$ mice the IGF-1 ${ }^{+}$cells appeared similar in number to untreated control mice. Scale bar, $15 \mu \mathrm{m}$.
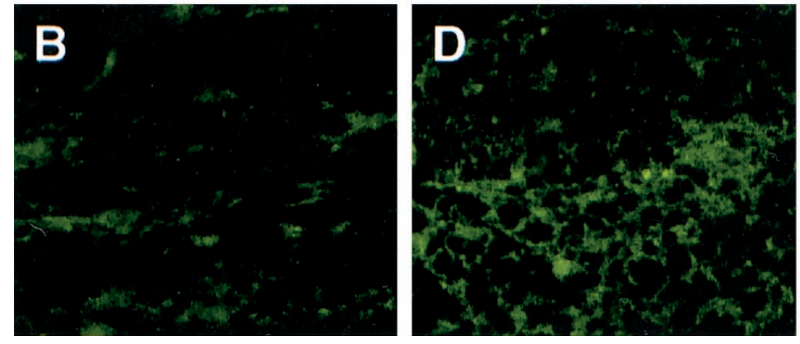

$\mathrm{NG} 2{ }^{+}$cells from three to four mice were quantified as described previously (Mason et al., 2000). Only positive-stained cells containing a nucleus were quantified.

Electron microscopy. Glutaraldehyde-fixed brain samples (three mice at each time point) were prepared as described previously (Coetzee et al., 1996). Coronal sections $(1 \mu \mathrm{m})$ were stained with toluidine blue, and the medial region of the corpus callosum was identified by light microscopy. The tissue was then trimmed and reoriented for thin sectioning so that cross sections of the corpus callosum could be examined by electron microscopy. Thin sections were cut, stained with uranyl acetate and lead citrate, and photographed, and then the electron micrographs were analyzed as described previously (Coetzee et al., 1996). Three hundred fibers $(0.3 \mu \mathrm{m}$ in diameter or greater) from each mouse were examined.

Protein analysis. Total protein was extracted from the forebrains of sham and treated mice as described previously (Ye et al., 1996). Protein concentrations were determined using a protein assay kit (Bio-Rad, Hercules, CA) with bovine serum albumin as the standard. Quantification of IGF-1 protein was determined by radioimmunoassay as described previously (Ye et al., 1996) using $20 \mu \mathrm{g}$ of total protein.

Statistical analysis. Statistical comparisons were made using a one-factor between-subjects ANOVA, followed by Tukey's test to assess significance among groups.

\section{RESULTS}

\section{Elevated expression of IGF-1 in transgenic mice}

To determine IGF-1 expression in the corpus callosum of wild-type mice, IGF-1 tg mice, and cuprizone-treated mice, IGF-1 RIA and immunostaining methods were used. IGF-1 protein levels were significantly $(p<0.01)$ greater in the forebrains of untreated control $I G F-1 \mathrm{tg}$ mice $(9.02 \pm 1.40 \mathrm{ng} / \mathrm{mg})$ compared with wild-type mice $(3.83 \pm 0.56 \mathrm{ng} / \mathrm{mg})$. This finding correlates with the presence of IGF-1 immunoreactivity within the corpus callosum of $I G F-1 \mathrm{tg}$ mice compared with the near absence of IGF- $1^{+}$cells in wild-type mice (Figs. $1 A, B)$. At 3 weeks of cuprizone exposure, IGF-1 immunoreactivity appeared to slightly increase relative to untreated controls in both the wild-type and $I G F-1 \mathrm{tg}$ mice (Fig. $1 C, D)$. By 5 weeks, intense IGF-1 immunoreactivity was elevated for the wild-type mouse compared with untreated control (Fig. 1, compare $A, E)$. This cellular expression pattern is consistent with our previous work demonstrating significant increase in IGF-1 protein by 4 and 5 weeks after the onset of mature oligodendrocyte apoptosis and depletion (Mason et al., 2000). In contrast, the corpus callosum of $I G F-1 \mathrm{tg}$ mice remained immunoreactive at 5 weeks but appeared to display levels similar to that observed in untreated $I G F-1 \mathrm{tg}$ mice (Fig. 1, compare $B, F$ ). Nonetheless, IGF-1 persists in the forebrains of $I G F-1 \mathrm{tg}$ mice throughout the 5 week cuprizone treatment period.

\section{Early recovery from acute demyelination in IGF-1 tg mice}

Exposing adult mice to cuprizone results in demyelination and then remyelination of the corpus callosum (Hiremath et al., 1998; Morell et al., 1998). We examined whether the presence of IGF-1
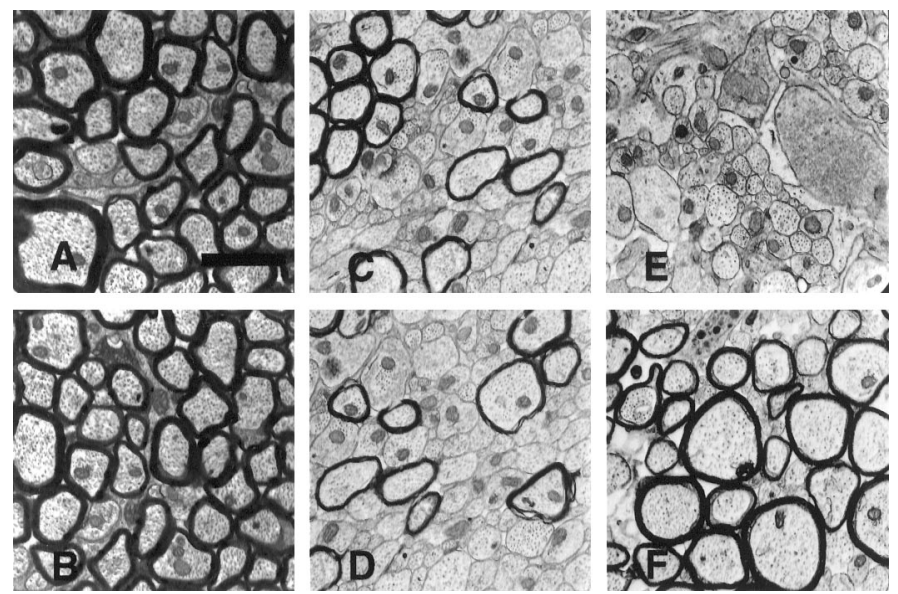

Figure 2. Demyelination and remyelination in the corpus callosum of $I G F-1$ tg and wild-type mice. Electron micrographs show that nearly all axons within the corpus callosum are myelinated in both the untreated wild-type $(A)$ and $I G F-1 \mathrm{tg}(B)$ mice. A large number of axons are demyelinated in both the wild-type $(C)$ and $I G F-1 \mathrm{tg}(D)$ mice exposed to cuprizone for 3 weeks. At 5 weeks, almost all of the axons are demyelinated in the wild-type mice $(E)$, whereas most of the axons are myelinated in the $I G F-1$ tg mice $(F)$. Scale bar, $1.2 \mu \mathrm{m}$.

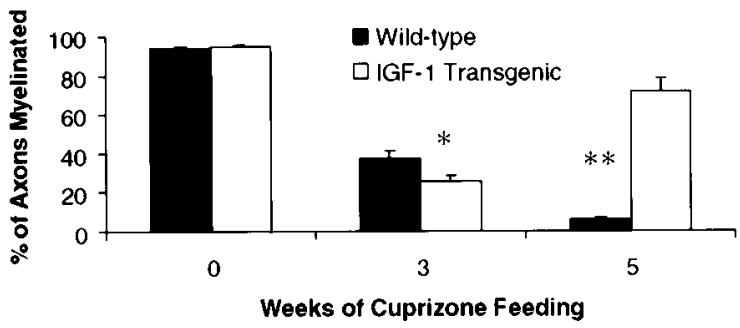

Figure 3. Percentage of myelinated axons within the corpus callosum of wild-type and $I G F-1$ tg mice. The percentage of myelinated axons within the corpus callosum of mice was determined by morphometric analysis of electron micrographs (300 axons per mouse were examined). The mean and SEM bars are plotted for each time point $(n=3)$ as examined in the wild-type (black bars) and IGF-1 tg (white bars) mice. $\left({ }^{*} p<0.01 ;{ }^{* *} p<\right.$ 0.001 when comparing wild-type mice with IGF-1 tg mice.)

during the first 5 weeks of cuprizone feeding could alter the demyelination process. Morphometric analysis demonstrated that nearly all axons within the corpus callosum were myelinated in both untreated control wild-type and $I G F-1 \mathrm{tg}$ mice (Figs. 2A,B, 3). After 3 weeks of exposure to cuprizone, the medial region of the corpus callosum was significantly demyelinated in both the wild- 

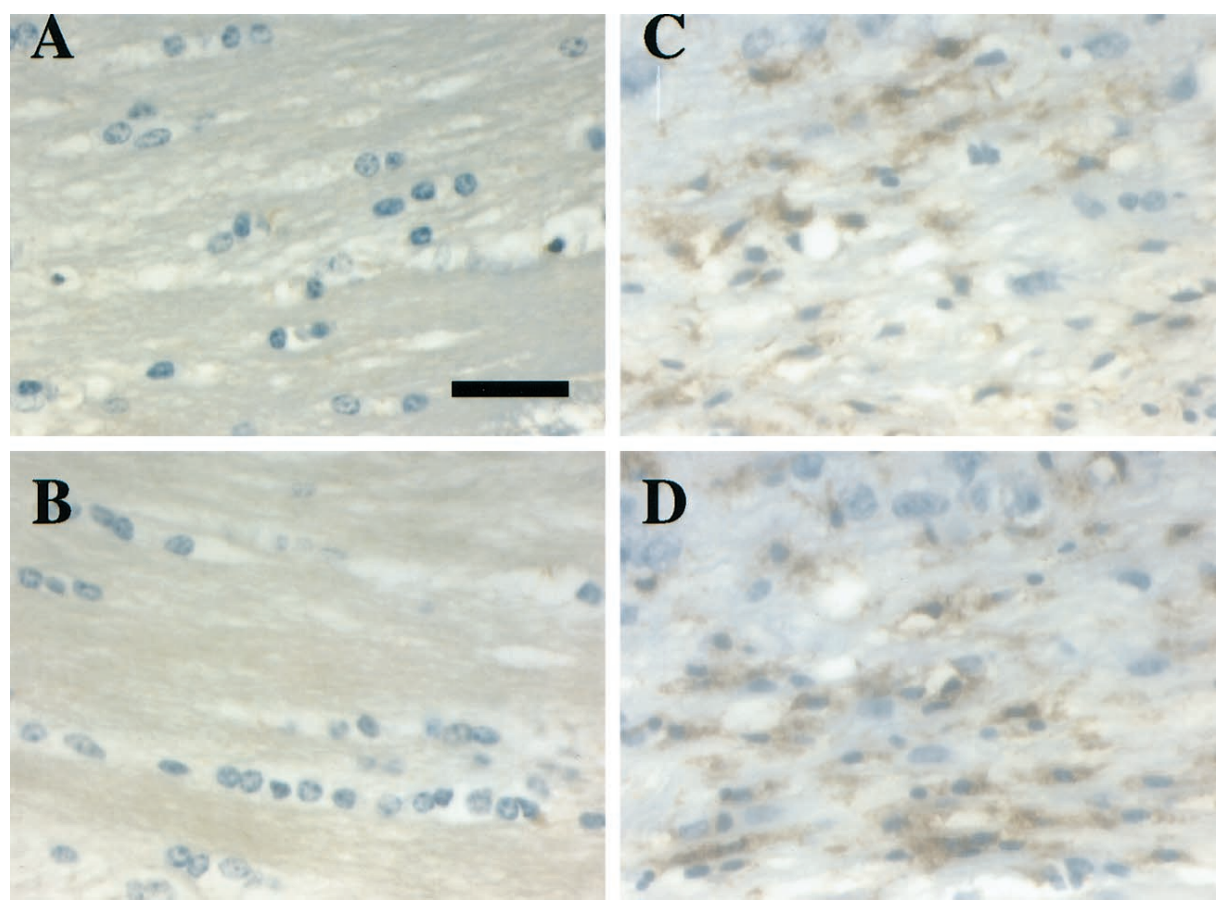

Figure 4. Microglia/macrophages associate with demyelination within the corpus callosum of cuprizone-treated wild-type and IGF-1 tg mice. Paraffin-embedded brain sections from wild-type $(A, C)$ and $I G F-1 \operatorname{tg}(B, D)$ mice were stained with RCA-1. No RCA-1 ${ }^{+}$cells were observed in the untreated control wild-type $(A)$ and $I G F-1$ tg $(B)$ mice. Large numbers of $\mathrm{RCA}^{-}{ }^{+}$cells (brown stain) were present in wild-type $(C)$ and IGF-1 tg $(D)$ mice fed cuprizone for 3 weeks. Scale bar, $25 \mu \mathrm{m}$.

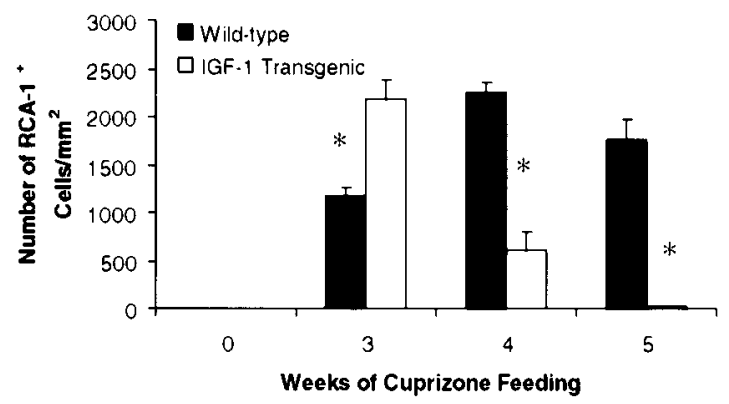

Figure 5. The accumulation of microglia/macrophages in the corpus callosum of wild-type and $I G F-1 \mathrm{tg}$ mice during cuprizone feeding. The number of RCA-1 ${ }^{+}$cells of wild-type mice (black bars) and IGF-1 tg mice (white bars) was quantitated in triplicate and plotted as mean \pm SEM $\left({ }^{*} p<\right.$ 0.001 when comparing wild-type mice and $I G F-1 \mathrm{tg}$ mice)

type and $I G F-1 \mathrm{tg}$ mice (Figs. 2C,D, 3). In wild-type mice, this continued until demyelination was nearly complete at week 5 (Figs. $2 E, 3)$. In contrast, the medial region of the corpus callosum was substantially remyelinated in the $I G F-1 \mathrm{tg}$ mice at 5 weeks (Figs. $2 F, 3$ ). These results suggest that the demyelinating insult was not as extensive in the $I G F-1 \mathrm{tg}$ mice and that the recovery from the demyelinating insult occurred earlier in these mice compared with wild-type mice.

\section{Microglia/macrophage accumulation corresponds to demyelination}

The accumulation of microglia/macrophages has been shown to be associated with demyelinating lesions in the CNS of adult mice (Hiremath et al., 1998). Microglia/macrophages (RCA- ${ }^{+}$cells) were very rare within the corpus callosum of untreated wild-type and $I G F-1 \mathrm{tg}$ mice (Figs. $4 A, B, 5$ ). By 3 weeks of cuprizone feeding, microglia/macrophages began to accumulate in large numbers within the corpus callosum of wild-type mice; however, even larger numbers accumulated within the demyelinating lesion in the $I G F-1$ tg mice (Figs. 4C,D, 5). By 5 weeks, very few RCA-1-positive cells were observed within the remyelinating corpus callosum of the $I G F-1$ tg mice, whereas a large number of these cells remained in the demyelinated corpus callosum of the wild-type mice at 5 weeks (Fig. 5). These results demonstrate that the presence of microglia/ macrophages correlates closely with the degree of demyelination within the corpus callosum.

\section{Survival of mature oligodendrocytes during demyelination}

The rapid recovery of the $I G F-1 \mathrm{tg}$ mice from demyelination led us to postulate that the mature oligodendrocytes in the $I G F-1 \mathrm{tg}$ mice may have survived the demyelinating insult. Before treatment, wild-type and $I G F-1 \mathrm{tg}$ mice appeared to have equal numbers of GST- $\mathrm{Pi}^{+}$oligodendrocytes within the corpus callosum (Figs. $6 A, B$, $7 A$ ). After 3 weeks of exposure to cuprizone, the number of GST- $\mathrm{Pi}^{+}$oligodendrocytes was reduced by $>50 \%$ in the wild-type mice (Fig. 6, compare $A$ with $C$ and Fig. 7A), and by week 5 they were severely depleted (Figs. $6 E, 7 A$ ). In contrast, only a slight reduction in the number of mature oligodendrocytes was observed in the $I G F-1 \mathrm{tg}$ mice (Fig. 6, compare $B$ with $D$ and Fig. $7 A$ ). The presence of IGF-1 appeared to allow for the rapid recovery of the mature oligodendrocyte population, because the number of GST$\mathrm{Pi}^{+}$oligodendrocytes returned to control levels by 4 and 5 weeks in the $I G F-1$ tg mice (Figs. $6 F, 7 A$ ). These results suggest that mature oligodendrocytes may be protected by the presence of IGF-1 before and during acute demyelination within the brain.

We have shown previously that the depletion of the GST-Pi ${ }^{+}$ population during cuprizone-induced demyelination is likely attributable to the apoptotic death of these cells (Mason et al., 2000). No apoptotic GST-Pi ${ }^{+}$oligodendrocytes were observed in untreated control wild-type or $I G F-1 \mathrm{tg}$ mice (Figs. $6 G, H, 7 B$ ). At 3 weeks of cuprizone exposure, a large number of GST-Pi ${ }^{+}$oligodendrocytes were undergoing apoptosis in the wild-type mice (Figs. 6I, 7B). This is consistent with previous findings that most apoptotic mature oligodendrocytes were observed between weeks 2 and 4 during exposure to cuprizone (Mason et al., 2000). In contrast, very few apoptotic oligodendrocytes were observed in the $I G F-1$ tg mice at week 3 (Figs. $6 J, 7 B$ ). These results suggest that the presence of IGF-1 within the CNS before acute demyelination appeared to keep mature oligodendrocytes from undergoing apoptosis.

\section{Absence of oligodendrocyte progenitors within the demyelinating corpus callosum of IGF-1 $\mathrm{tg}$ mice}

We have demonstrated previously that oligodendrocyte progenitors accumulate and differentiate within lesions that were depleted of preexisting mature oligodendrocytes, thereby leading to the repopulation of the mature oligodendrocytes (Mason et al., 2000). We speculated that the survival of the mature oligodendrocytes within the demyelinating lesion in the IGF-1 tg mice may inhibit the 

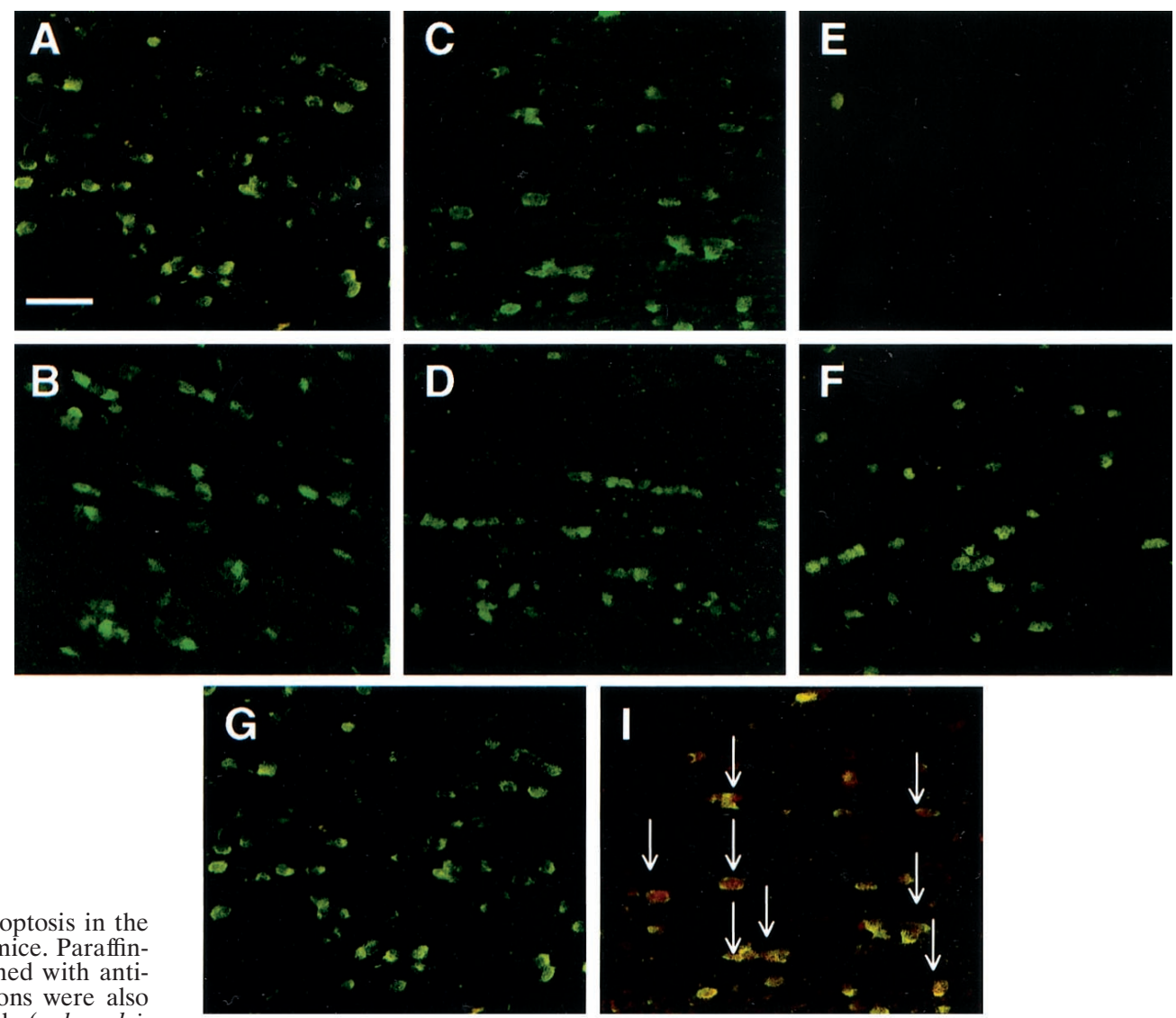

Figure 6. Mature oligodendrocytes and apoptosis in the corpus callosum of wild-type and $I G F-1 \mathrm{tg}$ mice. Paraffinembedded brain sections were immunostained with antiGST-Pi (green cells; $A-J$ ), and some sections were also examined for the presence of apoptotic cells (red nuclei; $G-J)$. Large numbers of GST-Pi ${ }^{+}$cells were observed in untreated control wild-type $(A)$ and $I G F-1 \operatorname{tg}(B)$ mice. At 3 weeks of exposure to cuprizone, there was a decrease in the number of GST-Pi ${ }^{+}$cells in wild-type mice $(C)$, whereas only a slight reduction was observed in the IGF-1 $\operatorname{tg}$ mice $(D)$. At 5 weeks, only a few GST-Pi ${ }^{+}$cells were observed in the wild-type mice $(E)$, whereas normal numbers of these cells were observed in the IGF-1 tg mice $(F)$. No apoptotic cells were observed in the untreated control wild-type $(G)$ and $I G F-1$ tg $(H)$ mice. At 3 weeks, a large number of apoptotic nuclei colocalized with GST-Pi ${ }^{+}$cells (arrow) in wild-type mice $(I)$, whereas no colocalization was observed in the $I G F-1 \mathrm{tg}$ mice $(J)$. Scale bar, $25 \mu \mathrm{m}$.
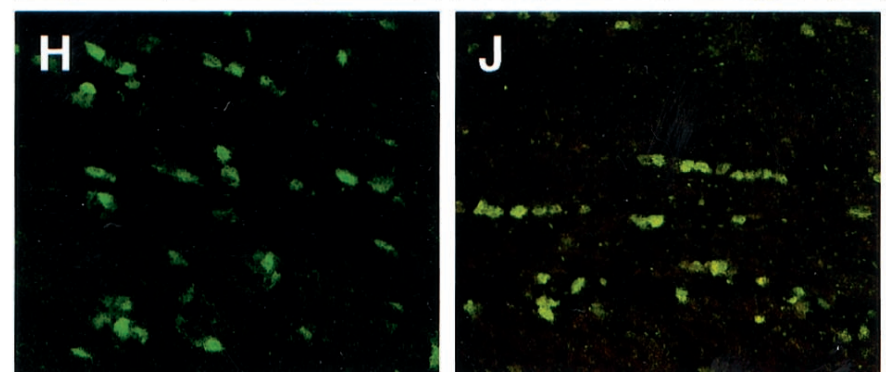

A

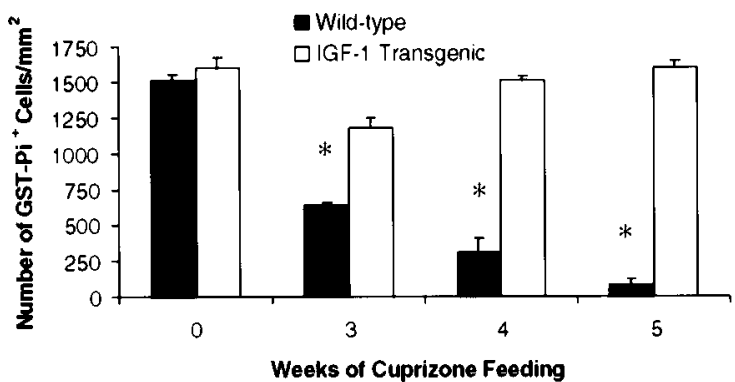

B

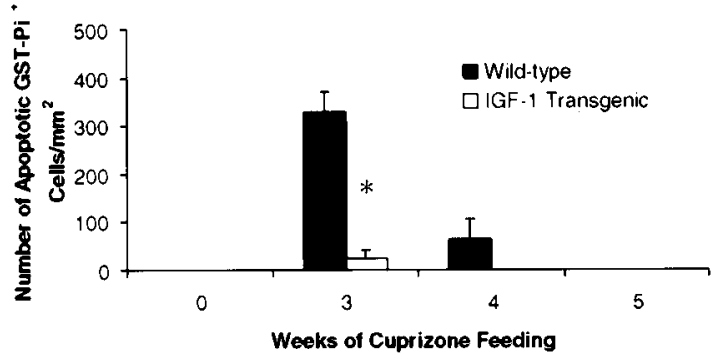

Figure 7. Survival of mature oligodendrocytes in $I G F-1$ tg mice. The number of GST-Pi ${ }^{+}$mature oligodendrocytes $(A)$ and the number of apoptotic mature oligodendrocytes $(B)$ were quantitated for wild-type mice (black bars) and IGF-1 tg mice (white bars) from triplicate samples and plotted as mean \pm SEM $\left({ }^{*} p<0.001\right.$ when comparing wild-type mice with IGF-1 tg mice).

ushering in of oligodendrocyte progenitors, although demyelination was evident. Before cuprizone treatment, few oligodendrocyte progenitors were present within the corpus callosum of untreated control wild-type and $I G F-1 \mathrm{tg}$ mice (Figs. $8 A, B, 9$ ). However, a large number of oligodendrocyte progenitors began to accumulate within the demyelinating corpus callosum in the wild-type mice at
3 weeks and continued to increase during demyelination through week 5 (Figs. 8C,9). In contrast, there was no accumulation of oligodendrocyte progenitors within either the demyelinating corpus callosum at week 3 or the remyelinating corpus callosum at weeks 4 and 5 in the $I G F-1$ tg mice (Figs. 8D, 9). These results suggest that the activation and accumulation of the oligodendro- 

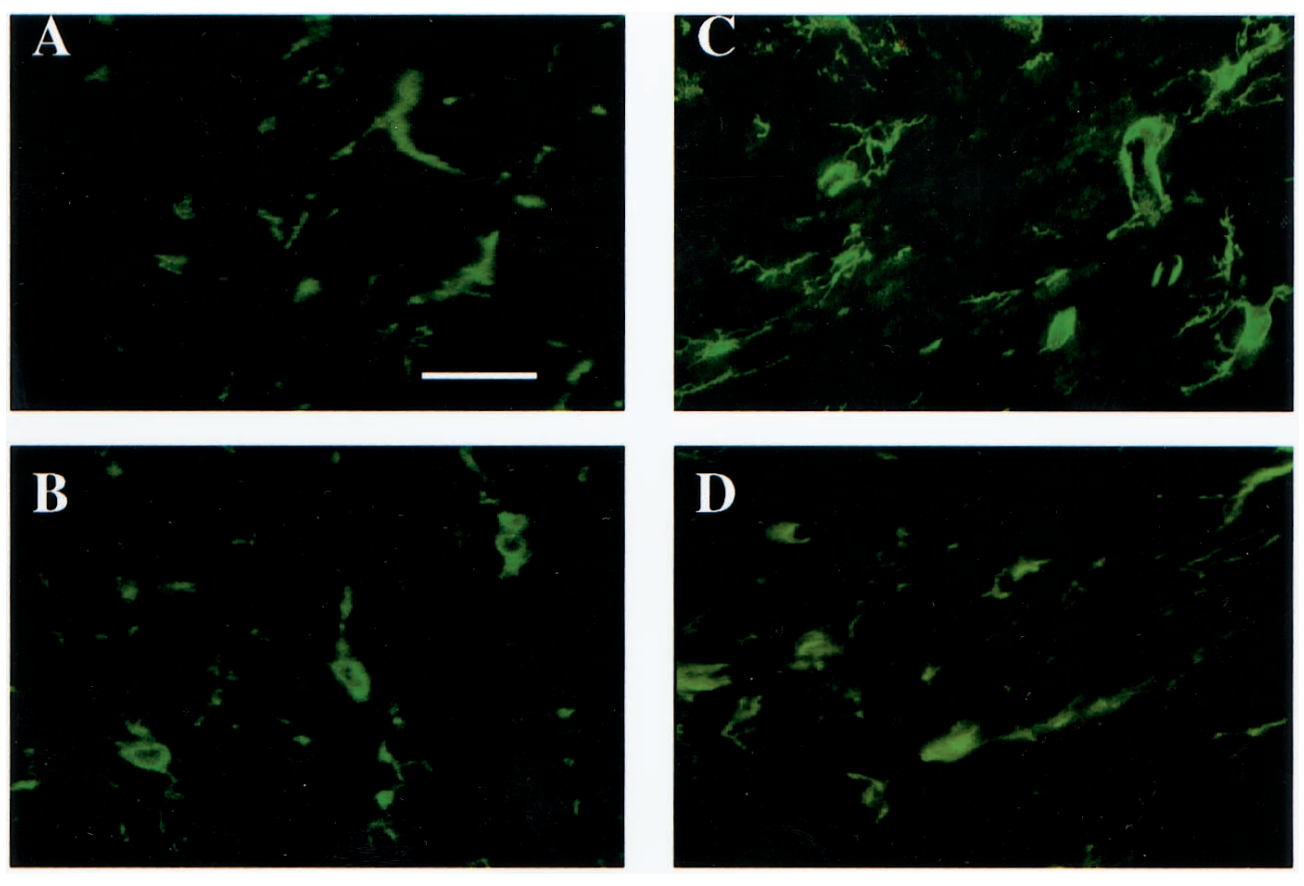

Figure 8. Accumulation of oligodendrocyte progenitors within the corpus callosum of wild-type but not $I G F-1 \mathrm{tg}$ mice during demyelination. Frozen brain sections from wild-type $(A, C)$ and $I G F-1 \mathrm{tg}$ $(B, D)$ mice were stained with the antiNG2 antibody (green). Few NG2 ${ }^{+}$cells were observed in the untreated control wild-type $(A)$ and $I G F-1 \operatorname{tg}(B)$ mice. Large numbers of $\mathrm{NG}^{+}$cells were present in wild-type mice exposed to cuprizone for 3 weeks $(C)$. Few $\mathrm{NG}^{+}{ }^{+}$cells were observed in the IGF-1 tg mice fed cuprizone for 3 weeks $(D)$. Scale bar, $10 \mu \mathrm{m}$.

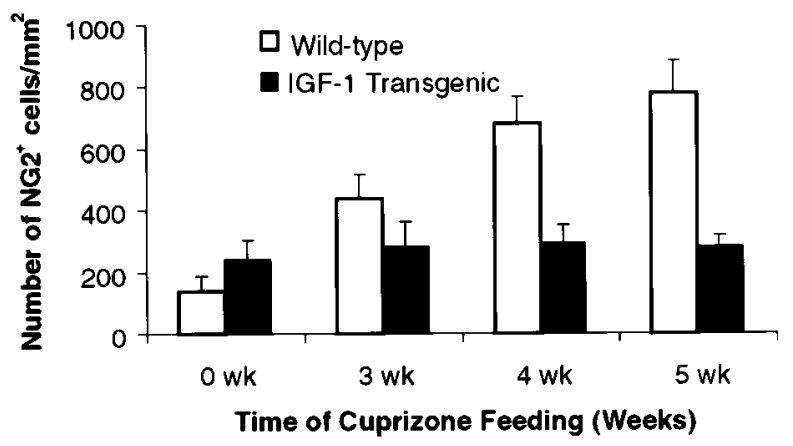

Figure 9. The accumulation of oligodendrocyte progenitors within the corpus callosum of wild-type and $I G F-1 \mathrm{tg}$ mice during cuprizone intoxication. The total number of $\mathrm{NG}_{2}{ }^{+}$cells was quantitated in the corpus callosum of wild-type mice (white bars) and $I G F-1$ tg mice (black bars) from triplicate samples and plotted as mean \pm SEM.

cyte progenitor population may be dependent on the absence of the mature oligodendrocyte population and not demyelination. In addition, these results also imply that the surviving mature oligodendrocytes in the $I G F-1$ tg mice most likely permit the early recovery from demyelination.

\section{DISCUSSION}

We have shown previously that mature oligodendrocytes are depleted by apoptosis during cuprizone-induced demyelination (Mason et al., 2000). In the present study, we have demonstrated several important findings. (1) The presence of IGF-1 before acute demyelination in the CNS appears to inhibit the apoptotic death and depletion of mature oligodendrocytes; (2) significant primary demyelination can occur despite survival of the mature oligodendrocyte population; (3) mature oligodendrocytes surviving a demyelinating insult appear to retain the ability to remyelinate; (4) the activation and accumulation of oligodendrocyte progenitors appear to occur in response to the absence of mature oligodendrocytes and not demyelination; and (5) the presence of microglia/macrophages correlates with each of the demyelination profiles for the wild-type and $I G F-1$ tg mice.

The present work showed that increased levels of IGF-1 did not inhibit microglia/macrophage accumulation and acute demyelination within the corpus callosum after exposure to cuprizone (Figs. $3,5)$. In fact, a greater number of microglia/macrophages and a slightly greater degree of demyelination were observed in the corpus callosum of $I G F-1 \mathrm{tg}$ mice at 3 weeks compared with the wild-type mice. Our observations are contrary to findings using the EAE model of demyelination (Liu et al., 1997). In EAE, the accumulation of macrophages and the induction of demyelination were blocked in mice given IGF-1 injections before a pathological insult. The authors attributed this pathological inhibition to the ability of IGF-1 to reduce the permeability of the blood-brain barrier (BBB) to immune effector cells, particularly T-cells, during EAE (Liu et al., 1995). This explanation may not be applicable because previous studies showed that the $\mathrm{BBB}$ is not compromised during cuprizone feeding (Kondo et al., 1987) and that T-cells play no significant role in cuprizone-induced demyelination (M. M. Hiremath, K. Suzuki, J.P.-Y. Ting. G. K. Matsushima, unpublished observations). Thus, effects of IGF-1 on the T-cell population and the BBB may not be relevant to cuprizone-induced demyelination.

The presence of IGF-1 early in the treatment period enhanced mature oligodendrocyte survival. However IGF-1 did not prevent acute demyelination. It is conceivable that the level of IGF-1 in the $I G F-1 \mathrm{tg}$ mice adequately protects the cell body of mature oligodendrocytes from apoptosis; yet, if it is perturbed enough, this may result in deterioration of the myelin sheath. Alternatively, during exposure to cuprizone, apoptosis of mature oligodendrocytes may be secondary to their lost contact with the axon, and IGF-1 may temporarily substitute for the axonal survival signal. These findings suggest that myelin pathology and mature oligodendrocyte death may be independent processes. Nonetheless, increased levels of IGF-1 appear to have a profound impact on the remyelination process. Demyelination within wild-type mice progressed and then peaked at 5 weeks of cuprizone feeding (Figs. 2C,E, 3), whereas remyelination was observed after 4 and 5 weeks in the IGF-1 tg mice (Figs. 2D,E, 3). These results support a controversial issue that the surviving mature oligodendrocytes are capable of remyelination. In addition, these finding are consistent with previous observations in EAE in which systemic injections of IGF-1, introduced after lesion formation, resulted in reduced clinical deficits and lesion severity while also upregulating the synthesis of myelin proteins (Yao et al., 1995, 1996). This rapid recovery may have been caused by an increase in myelin synthesis, the survival of the mature oligodendrocyte population, or a combination of both.

Previous studies have recognized mature oligodendrocyte depletion as the primary factor in the formation of chronic demyelinating lesions (Johnson and Ludwin, 1981; for review see Raine, 1997; 
J. L. Mason, Hostettler JD, Morell P, Suzuki K, Matsushima GK unpublished observations). We have demonstrated that the depletion of mature oligodendrocytes during cuprizone-induced demyelination is attributable to the apoptotic death of these cells (Mason et al., 2000). The present work, which demonstrates the ability of IGF-1 to inhibit mature oligodendrocyte apoptosis in vivo (Fig. $7 B$ ), is consistent with previous in vitro studies (Cho et al., 1997; Ye and D'Ercole, 1999). Although no or rare apoptotic oligodendrocytes were observed, it is plausible that apoptotic cells escaped our method of detection or sampling interval that could have accounted for the slight reduction in the mature oligodendrocyte population in the IGF-1 tg mice at 3 weeks. Similarly, we cannot rule out the possibility that this reduction in mature oligodendrocytes could be a consequence of necrosis and/or dedifferentiation. However, our data suggest that inhibiting the death of mature oligodendrocytes not only prevents their depletion, but it may prevent the formation of chronic plaques in demyelinating diseases such as multiple sclerosis.

$I G F-1$ tg mice when compared with wild-type mice displayed dramatic differences in oligodendrocyte progenitor recruitment after demyelination. Wild-type mice showed an accumulation of oligodendrocyte progenitors within the demyelinating lesion (Fig. 8) consistent with previous observations in the adult rodent CNS (Gensert and Goldman, 1997; Keirstead et al., 1998; Mason et al., 2000). In contrast, there was no accumulation of oligodendrocyte progenitors within the demyelinating lesion in the $I G F-1 \mathrm{tg}$ mice where mature oligodendrocytes remained present (Fig. 8). These results suggest that the activation and/or accumulation of oligodendrocyte progenitors within a demyelinating lesion may be in response to the death or absence of the mature oligodendrocyte population as observed in wild-type mice and not caused by demyelination alone. Furthermore, the surviving mature oligodendrocytes within the demyelinated lesion most likely are responsible for the early remyelination observed in the IGF-1 tg mice. These results imply that perturbed mature oligodendrocytes regain their ability to myelinate when protected by the presence of IGF-1. If IGF-1 is not present at sufficient levels, as appears to be the case in wild-type mice, before treatment or during the first 2 weeks of exposure to cuprizone, then mature oligodendrocytes may become susceptible to an apoptotic death, which eliminates their role in remyelination. In wild-type mice, exposure to cuprizone results in oligodendrocyte death that progresses to their near complete depletion from the corpus callosum, and remyelination is primarily caused by the recruitment of oligodendrocyte progenitors that eventually repopulate and differentiate within the lesions (Mason et al., 2000). Our work here implies that mature oligodendrocytes are capable of substantial remyelination of demyelinated axons. Combined with previous in vitro (Cho et al., 1997; Ye and D’Ercole, 1999) and in vivo (Yao et al., 1996; Liu et al., 1997) studies, the present work strengthens the support for studying the therapeutic merits of growth factors such as IGF-1 in demyelinating diseases.

\section{REFERENCES}

Barres BA, Schmid R, Sendnter M, Raff MC (1993) Multiple extracellular signals are required for long-term oligodendrocyte survival. Development 118:283-295.

Cho KH, Kim MW, Kim SU (1997) Tissue culture model of Krabbe's disease: psychosine cytotoxicity in rat oligodendrocyte cultures. Dev Neurosci 19:321-327.

Compston A (1996) Remyelination of the central nervous system. Mult Scler 1:388-392.
Coetzee T, Fujita N, Dupree J, Shi R, Blight A, Suzuki K, Suzuki K, Popko B (1996) Myelination in the absence of galactocerebroside and sulfatide: normal structure with abnormal function and regional stability. Cell 86:209-219.

D'Ercole AJ, Ye P, Calikoglu AS, Gutierrez-Ospina G (1996) The role of the insulin-like growth factors in the central nervous system. Mol Neurobiol 13:227-255.

Gensert JM, Goldman JE (1997) Endogenous progenitors remyelinate demyelinated axons in the adult CNS. Neuron 19:197-203.

Hiremath MM, Saito Y, Knapp GW, Ting JP-Y, Suzuki K, Matsushima GK (1998) Microglial/macrophage accumulation during Cuprizone-induced demyelination in C57BL/6 mice. J Neuroimmunol 92:38-49.

Johnson ES, Ludwin SK (1981) The demyelination of recurrent demyelination and remyelination of axons in the central nervous system. Acta Neuropathol 53:93-98.

Keirstead HS, Levine JM, Blakemore WF (1998) Response of the oligodendrocyte progenitor cell population (defined by NG2 labeling) to demyelination of the adult spinal cord. Glia 22:161-170.

Kondo A, Nakano T, Suzuki K (1987) Blood-brain barrier permeability to horseradish peroxidase in twitcher and cuprizone-intoxicated mice. Brain Res 425:186-190.

Liu X, Yao D-L, Webster HD (1995) Insulin-like growth factor I treatment reduces clinical deficits and lesion severity in acute demyelinating experimental autoimmune encephalomyelitis. Mult Scler 1:2-9.

Liu X, Linnington C, Webster HD, Lassmann S, Yao D-L, Hudson LD, Werkle H, Kreutzberg GW (1997) Insulin-like growth factor-1 treatment reduces immune cell responses in acute non-demyelinative experimental autoimmune encephalomyelitis. J Neurosci Res 47:531-538.

Mason JL, Jones JJ, Taniike M, Morell P, Suzuki K, Matsushima GK (2000) Mature oligodendrocyte apoptosis precedes IGF-1 production and oligodendrocyte progenitor accumulation and differentiation during demyelination/remyelination. J Neurosci Res, in press.

Morell P, Barrett CV, Mason JL, Toews AD, Hostettler JD, Knapp GW, Matsushima GK (1998) Gene expression in brain during Cuprizoneinduced demyelination and remyelination. Mol Cell Neurosci 12:220-227.

Mozell RL, McMorris FA (1991) Insulin-like growth factor I stimulates oligodendrocyte development and myelination in rat aggregate cultures. J Neurosci Res 30:382-390.

Pender MP, Nguyen KB, McCombe PA, Kerr JFR (1991) Apoptosis in the nervous system in experimental allergic encephalomyelitis. J Neurol Sci 104:81-87.

Raine CS (1997) The Norton Lecture: a review of the oligodendrocyte in the multiple sclerosis lesion. J Neuroimmunol 77:135-152.

Sidman RL, Abervine JB, Pierec ET (1971) Atlas of the mouse brain and spinal cord. Cambridge, MA: Harvard UP.

Taniike M, Mohri I, Eguchi N, Irikura D, Urade Y, Okada S, Suzuki K (1999) An apoptotic depletion of oligodendrocytes in the twitcher, a murine model of globoid cell leukodystrophy. J Neuropathol Exp Neurol $58: 644-653$.

Werther GA, Russo V, Baker N, Butler G (1998) The role of the insulinlike growth factor system in the developing brain. Horm Res 49[Suppl 1]:37-40.

Yao D-L, West NR, Bondy CA, Brenner M, Hudson LD, Zhou J, Collins GH, Webster HD (1995) Cryogenic spinal cord injury induces astrocytic gene expression of insulin-like growth factor 1 and insulin-like growth factor binding protein 2 during myelin regeneration. J Neurosci Res 40:647-659.

Yao D-L, Liu X, Hudson LD, Webster HD (1996) Insulin-like growth factor-1 given subcutaneously reduces clinical deficits, decreases lesion severity and upregulates synthesis of lyelin proteins in experimental autoimmune encephalomyelitis. Life Sci 58:1301-1306.

Ye P, Carson J, D'Ercole AJ (1995) In vivo actions of insulin-like growth factor-1 (IGF-1) on brain myelination: studies of IGF-1 and IGF binding protein-1 (IGFBP-1) transgenic mice. J Neurosci 15:7344-7356.

Ye P, Xing Y, Dai Z, D'Ercole AJ (1996) In vivo actions of insulin-like growth factor-1 (IGF-1) on cerebellum development in transgenic mice: evidence that IGF-1 increases proliferation of granule cell progenitors. Dev Brain Res 95:44-54.

Ye P, D'Ercole AJ (1999) Insulin-like growth factor I protects oligodendrocytes from tumor necrosis factor- $\alpha$-induced injury. Endocrinology 140:3063-3072. 